\title{
Chemometrics in the Age of Intelligent Chemical Instruments
}

\section{Peter de Boves Harrington ${ }^{1}$}

Published online: 20 December 2018

(c) The Nonferrous Metals Society of China 2018

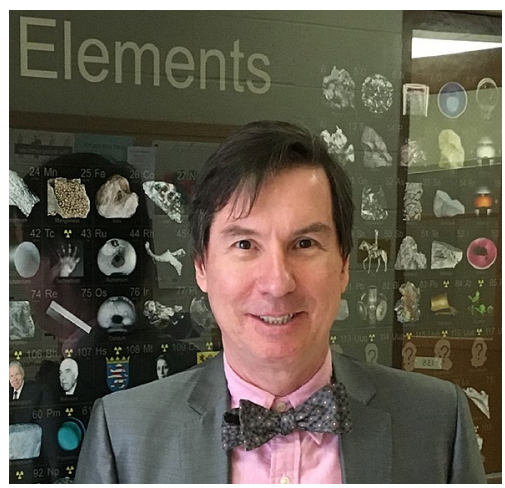

We are presently living through a dawning of a new era for which artificial intelligence (AI) will affect every aspect of our daily life. Already, smartphones contain digital personal assistants that can translate our voice commands into digital signals that can control our phones or purchase items for us through the internet. These advances in our personal technology can also be used for analysis and testing.

All scientists conduct experiments and most of them involve chemical systems. Analytical chemistry is the discipline of making measurements of chemical systems and because most scientific measurements involve some aspect of chemistry, analytical chemistry is central to all sciences. Central to the traditional sub-disciplines of analytical chemistry, quantitative analysis, spectrochemistry, separations, and electrochemistry, is chemometrics. Chemometrics is the science of maximizing the information gained from a chemical experiment. It includes the design of experiments, statistical inference, and knowledge extraction from data.

Peter de Boves Harrington

harringp@ohio.edu

1 Clippinger Laboratories, Department of Chemistry and Biochemistry, Center for Intelligent Chemical Instrumentation, Ohio University, Athens, OH 45701-2979, USA
By automating the chemometrics, one can devise intelligent chemical instruments.

This special issue contains contributions from some of the stars in the field of chemometrics reporting on some of the pertinent applications. The paper by Zewei Chen et al. deals with chemotyping botanical medicines by a high-throughput device. The chemotype coupled to chemometrics furnishes a method for rapidly authenticating complex materials for pharmacological activity, provenance, or quality. The paper by Yifei Wang and Pei Chen is using fuzzy chromatography for plant-based metabolomics. Douglas Routledge demonstrates that common components analysis, a modern approach for dimensionality reduction and mathematical chromatography to extract pure signals from mixed ones, is superior to the traditional method of principal component analysis and more recent independent components analysis. Yun Shu and Royston Goodacre provide a review of model validation which is important for smart instruments to evaluate how well that they are operating (sometimes called meta-thinking). Md. Aminul Islam Prodhan et al. devised a method to convert two-dimensional chromatographic retention indices into useful information. Sergey Kucheryavski demonstrated the benefits of classification and regression trees and forests for solving problems in classification and calibration. Siewert Hugelier et al. discuss strategies for efficient processing of hyperspectral images (images that comprise a stochastic fluorescent profile for each picture element) to enhance super-resolution microscopy.

In summary, the field of chemometrics is vibrant with developments that will yield intelligent chemical instrumentation. On behalf of the authors, we thank the new Journal of Analysis and Testing to provide a venue for showcasing ground-breaking research in Analytical Chemistry. We also thank the reviewers whose helpful and timely comments make this special issue possible.

Professor Peter de Boves Harrington graduated from Randolph-Macon College in 1980 with a Baccalaureate of Science in Chemistry. After which, he worked as flavor chemist for Nabisco from 1980 to 1982. In 1988, Pete graduated from the University of North Carolina-Chapel Hill. His 
Ph.D. dissertation is titled Applications of Pattern Recognition and Artificial Intelligence to Some Problems in Analytical Chemistry. He developed pattern recognition methods for identifying bacteria from their pyrolysis-mass spectra and created the DOS-based software platforms Resolve and Presager, when he was a Research Assistant Professor from 1987 to 1989 at the Colorado School of Mines. Pete joined the faculty at Ohio University in 1989 as an Assistant Professor. In 1992, he founded the Center for Intelligent Chemical Instrumentation. He has over 200 publications and has made over 300 scientific presentations around the world. Recently,
Pete won the Ohio University College of Arts and Sciences Outstanding Faculty Research Award and the Eastern Analytical Symposium Award for Outstanding Achievement in Chemometrics. Pete is the Director of the Ohio University Center for Intelligent Chemical Instrumentation and is a Fellow of the American Academy of Forensic Sciences and the North American Academy of Sciences. Currently, Pete's research focuses on the development and coupling of artificial intelligence to chemotyping by spectrometry of botanical medicines and foods. 